\title{
X-ray Evolution of SNR 1987A: The Radial Expansion
}

\author{
Judith L. Racusin ${ }^{1}$, Sangwook Park ${ }^{1}$, Svetozar Zhekov ${ }^{2,3}$, David N. Burrows ${ }^{1}$, Gordon P. \\ Garmire $^{1}$, Richard McCray ${ }^{2}$
}

\begin{abstract}
We present the evolution of the radial expansion of SNR 1987A as measured using Chandra X-ray observations taken over the last 10 years. To characterize the complex structure of the remnant and isolate the expansion measurement, we fit the images to several empirical models including: a simple circular torus, a torus with bilateral lobes, and a torus with four tangentially extended lobes. We discuss the results of this measure in the context of the overall evolution of the supernova remnant, for which we believe we have measured the end of the free expansion phase and its transition to the adiabatic phase (at least along the equatorial ring). The timing of this event is in agreement with early predictions of the remnant evolution.
\end{abstract}

\section{Introduction}

Supernova 1987A is the nearest and consequently the most extensively studied supernova (SN) in modern astronomy. While it has led to important insights into progenitor systems and supernova evolution, it is by no means a typical characteristic event. The progenitor was seen in archival plates as blue supergiant $S k$-69 202 (White \& Malin 1987) rather than the expected red supergiant progenitor of a Type II SN. The extent of the stellar winds from both the red and blue supergiant phases suggest that the system transitioned from a red supergiant sometime in the last $\sim 40,000$ years (Arnett et al. 1989). This transition may explain some of the unusual properties of the supernova remnant (SNR).

The ring-like structure visible and evolving over time in X-ray, optical, and radio observations was first discovered to have "clumpy" structure using the high-resolution Hubble

\footnotetext{
${ }^{1}$ Department of Astronomy \& Astrophysics, The Pennsylvania State University, University Park, PA 16802

${ }^{2}$ JILA, University of Colorado, Box 440, Boulder, CO, 80309

${ }^{3}$ On leave from Space Research Institute, Sofia, Bulgaria
} 
Space Telescope images (Plait et al. 1995). The many spots seen in the optical appear to correspond to the less resolved soft X-ray spots. The X-ray and radio image structures are much simpler than the complex triple-ring structure seen in optical observations (Burrows et al. 1995); the X-rays and radio emission are only detected from the central inner ring. This ring appears to be a structure of hot gas that is flattened into a round disk. It appears elliptical due to its inclination, is gradually brightening in all bands, shows increasing structural details, and is expanding radially. The optical emitting region of the inner ring is slightly outside that of the X-ray and radio emitting regions. The soft X-ray emission from SNR 1987A is thought to be produced by shocked hot gas that lies between the forward and reverse shocks of the SN blast wave, with the localized spots of emission around the inner ring being due to regions of enhanced density protruding from the inner surface of the circumstellar ring (Park et al. 2002; Sugerman et al. 2002). The inner and outer rings are thought to be produced by asymmetric stellar winds shed at different stages in the evolution of the massive progenitor star (Luo \& McCray 1991; Chevalier \& Dwarkadas 1995).

The soft X-ray emission has been increasing in intensity as a result of the blast wave shocking and continually sweeping up the surrounding circumstellar material. The structure of the remnant has evolved from emission largely contained in these small regions to overall brightening of the ring during the progressive observations of the resolved remnant. As the blast wave lights up the dense inner ring, the X-ray spectrum may become dominated by the density structure of the ring itself (Park et al. 2006a). Figure 1 shows the $0.3-8.0 \mathrm{keV}$ Chandra images at each epoch (Table 1), showing how the X-ray emission has transitioned in recent years from being dominated by the shocked gas in the dense hot spots to being dominated by the gas between the forward and reverse shocks (McCray 2003).

The ROSAT X-ray light curve increased linearly for approximately 10 years after the SN explosion. Later Chandra observations indicated that there was a deviation beginning in $\sim 1997$ ( $\sim 3700$ days after the SN) from the ROSAT linear extrapolation of the flux, which results in a nearly exponential increase in the soft X-ray light curve (Figure 2). This transition was temporally coincident with the appearance of the first optical spot (Park et al. 2002; Sonneborn et al. 1998). This rapid flux increase was followed by an upturn in the X-ray light curve in 2004 ( 6200 days after the SN), suggesting that the blast wave had reached the main body of the inner ring. This brightening was accompanied by substantial softening of the overall X-ray spectrum, brightening of hot spots in the ring, filling in of the X-ray ring, and a decrease in radial expansion velocity of the ring.

In this paper we describe a new method for measuring the size of the remnant from the $\mathrm{X}$-ray images, and use that to put it in the context of the structure and overall evolution of the system. We describe in $\$ 2$ the predictions in the literature prior to our Chandra 
campaign, the observations in \$3, our radial expansion measurement and image models in $\$ 4$, the results in $\$ 5$, the discussion in $\$ 6$, and our conclusions in $\$ 7$.

\section{Predictions}

Early observations of the circumstellar ring and measurements of the ejecta velocities prompted predictions for when the blast wave would encounter the inner ring (Chevalier \& Liang 1989; Luo \& McCray 1991). This encounter was predicted to occur between 8 and 16 years after the SN and would result in dramatic re-brightening as a result of the strong shocks produced by the blast wave interacting with the inner ring. This prediction was later revised when Chevalier \& Dwarkadas (1995) suggested that an H II region may exist inside the inner circumstellar ring. It is needed to reconcile the expected low mass loss rate $\left(\dot{M} \sim 7.5 \times 10^{-8} \mathrm{M}_{\odot} \mathrm{yr}^{-1}\right)$ of the blue supergiant progenitor with the early radio free-free absorption and the X-ray emission levels. The H II region was probably formed in material swept up by the red supergiant wind that was ionized by the blue supergiant star. This has the effect of delaying the date of the encounter with the inner ring to year $2005 \pm 3$ because the blast wave is slowed as it swept up this additional material.

Borkowski et al. (1997) modeled the SN blast wave impact with the inner circumstellar ring and the resulting reverse shock. They predicted that the impact would be characterized by a gradual increase in X-ray luminosity. As the shock front engulfed the inner ring, it was expected to gradually brighten by as much as 3 orders of magnitude in the optical, UV, and $\mathrm{X}$-ray. The X-ray spectrum would also change depending on the detailed shock structure of the interaction with the inner ring. The overall soft X-ray flux evolution is consistent with these predictions, showing a steep increase in the light curve since $\sim 2000$ (see Figure 2). However, these predictions were made before the emergence of the first optical spot, and therefore could not have accounted for how the higher density protrusions would affect the observational behavior.

After $\sim 1997(\sim 3700$ days after the SN) the soft X-ray light curve began to deviate from the linear increase observed for the prior 10 years. This brightening was initially confined to the localized hot spots evident in HST images in the northeastern and southeastern regions of the inner ring. As more optical hot spots appeared, the X-ray emission also spread around the ring and the X-ray luminosity increased dramatically. The brightening of the entire ring of SNR 1987A appears to have begun in early 2004 and the events predicted

are underway. This is evident in the brightening in X-rays (Park et al. 2005), infrared (Bouchet et al. 2006), and optical (McCrav 2005), the continuous softening of the X-ray spectrum (Park et al. 2006b; Zhekov et al. 2005), deceleration of the radial expansion of the 
SNR (Park et al. 2004; Zhekov et al. 2005), and slower shock velocities from line broadening (Michael et al. 2002; Dewey et al. 2008; Zhekov et al. 2009).

\section{Chandra Observations and Data Reduction}

We have observed SNR 1987A with Chandra on 23 occasions between 1999 October and 2009 January, and our monitoring observations continue at approximately 6 month intervals. Eighteen of the observations were carried out using the Advanced CCD Imaging Spectrometer (ACIS) back-illuminated S3 CCD. ACIS alone provides high-resolution images with moderate non-dispersive energy resolution. When used in conjunction with one of the gratings, either the High Energy Transmission Grating (HETG) or the Low Energy Transmission Grating (LETG), ACIS provides high quality spectra and an undispersed zeroth-order image with moderate energy resolution. Six of our observations used the gratings: HETG (1999 October and 2007 April, 2008 July, 2009 January, (Burrows et al. 2000; Michael et al. 2002; Dewey et al. 2008)) and LETG (2004 September and 2007 September, (Zhekov et al. 2005, 2006, 2009)). In the standard LETG configuration, the long-wavelength oxygen lines are positioned on the sensitive ACIS-S3 chip, which consequently places the zeroth order image 1.5' off-axis, blurring it by Chandra's off-axis Point Spread Function (PSF). This blurring degrades the image, and we therefore exclude the LETG observations from our measurement of the radial expansion rate. The HETG, however, does not suffer from this effect. The differences in the images between the bare-ACIS and the zeroth-order HETG have not been calibrated, though no significant systematic effects (as seen with the LETG) are expected. The first HETG observation is also our first monitoring observation, therefore it is an important reference point for our analysis. The deep HETG observation taken in 2007 was not part of our regular monitoring observations and was performed in between regularly scheduled monitoring observations. As the remnant brightens, we have recently taken calibration observations comparing the bare-ACIS and HETG zeroth-order images (in 2008-7 and 2009-1). In order to avoid photon pipe-up, we will transition future observations to HETG only. These closely spaced HETG observations to monitoring ACIS observations revealed consistent radii between the ACIS-only and HETG measurements. They do not add new information to this study and therefore do not have a strong affect on the radial expansion velocity fits. We exclude all gratings observations except the 1999 October HETG observation from the rest of radial expansion analysis presented in this paper. The details of the 19 Chandra observations used in this work are listed in Table 1 .

The technique used to create our images is the same as was used in our previous works

(Burrows et al. 2000; Park et al. 2002, 2004, 2006a, b). The event data used to create the 
images were filtered to use the energy range $0.3-8 \mathrm{keV}$, and ASCA grades 02346. Flaring pixels were removed. We corrected for the charge transfer inefficiency (CTI) using the

methods developed by Townsley et al. (2000). The small angular extent of the remnant $\left(\sim 1^{\prime \prime}\right.$.6) makes any image analysis difficult even with the superb spatial resolution of Chandra (ACIS CCD pixel size $\sim 00^{\prime \prime} 492$ ). To better sample the image, we applied the sub-pixel resolution method developed by Tsunemi et al. (2001) and Mori et al. (2001). This technique uses the feature that information can be obtained on the location within the pixel where the photon was absorbed by examining the distribution of the events split between pixels. The morphology of the splitting can be traced to a centroid within the pixel for events spread across more than 2 pixels. This technique improves the angular resolution of the images by $\sim 10 \%$.

Several other factors also help to improve the image resolution with the use of the subpixel resolution technique. The choice of using one of the ACIS back-illuminated CCDs leads to a larger fraction of split-events. The back illuminated chips are also less affected by the poor charge transfer inefficiency for observations obtained after the particle damage in September 1999 (Townsley et al. 2000). Another source of better resolution comes from the intentional dithering motion of the observatory which causes a target image to move across the CCD surface forming a Lissajous pattern. This leads to more opportunities of forming split-pixel events in different areas of the image. Standard processing adds a randomization of event positions within each pixel to prevent marked pixelization of the images. We turn off this randomization in the data processing to prevent any loss of positional information.

To further improve the effective angular resolution of the images, we deconvolve them with a maximum likelihood algorithm (Richardson 1972; Lucy 1974) using the on-axis detector PSF. We used a scale of $00^{\prime \prime} 125$ sky pixels and then smoothed the images by convolving with a Gaussian $(\sim 0$ '.1 FWHM). The resulting $0.3-8.0 \mathrm{keV}$ broadband images of SNR 1987A from each of the epochs of our observations are shown in Figure 1.

\section{Radial Expansion}

Previous works to model the X-ray radial expansion rate of SNR 1987A (Park et al. 2002, 2004) used a simple Gaussian model to fit the radially averaged profile. This approach yielded an expansion velocity estimate of $4000-5000 \mathrm{~km} \mathrm{~s}^{-1}$. The utility of this simple method was limited by the loss of information on the detailed features such as the lobes, the overall asymmetric intensity, as well as ellipticity and inclination of the inner ring. To more accurately quantify the radial expansion, we developed a more realistic image model to estimate the SNR's radius. 
The goal for our empirical models is to characterize the Chandra X-ray images in a more realistic way and in a similar spirit as those of the radio studies (Gaensler et al. 1997; Manchester et al. 2002, 2005; Ng et al. 2008) in order to obtain a measurement for the radial expansion of the remnant. The inner ring is inclined by $43^{\circ}$ to the line of sight (Crotts \& Heathcote 1991). The bulk of the X-ray emission originates from the "disk" con-

taining the inner ring, rather than from a spherical volume (Zhekov et al. 2005). The X-ray morphology of SNR 1987A appears to be an elliptical ring with 3-4 enhanced lobes (Figure 11). The real number of X-ray spots is likely larger, but the Chandra spatial resolution prohibits the distinguishing of finer image details. To remove the apparent ellipticity prior to fitting the observed images with our models, we deproject the images by the $43^{\circ}$ inclination. After the deprojection, the images are approximately circular (e.g., Figure $3 \mathrm{a}$ and $3 \mathrm{~b}$ ). We fit three simple empirical models to the image data in an attempt to measure the radial expansion velocity, with the latter two models removing the biases created by the brightening hot spots.

\subsection{Torus Only Model}

The torus model is the simplest of the three models. It assumes that the images can be approximated by a circularly symmetric smooth ring with a peak radius of $r_{0}$ and a Gaussian width of $\sigma_{r_{0}}$, with the following form:

$$
\begin{gathered}
r=\left[\left(x-x_{0}\right)^{2}+\left(y-y_{0}\right)^{2}\right]^{1 / 2} \\
M_{\text {torus }}=N_{0}+N_{r} \exp \left[\frac{-\left(r-r_{0}\right)^{2}}{2 \sigma_{r_{0}}^{2}}\right]
\end{gathered}
$$

where $x$ and $y$ are the coordinates of each pixel in the model image, $x_{0}$ and $y_{0}$ are the offsets of the ring center from the image center, $N_{0}$ is the background image level, $N_{r}$ is the normalization, and $M_{\text {torus }}$ is the resulting model image. An example of a fit to this model is shown in Figure 3 c.

\subsection{Bilateral Lobes + Torus Model}

This model consists of the torus as described in \$4.1 plus 2 bilateral lobes. The addition of bilateral lobes approximates the morphology of the radio remnant (Gaensler et al. 1997; Manchester et al. 2002, 2005; $\mathrm{Ng}$ et al. 2008). The morphology of the X-ray remnant in the earliest epochs resembled that of the radio remnant with emission approximated by opposite bilateral regions of increased brightness. As the X-ray spots have brightened, additional 
lobes are required to adequately model the images. Therefore, this model works best in the early epochs and poorly in late ones. We required the lobes to be positioned with one on each the East and West side of the remnant and allow them to vary in intensity $\left(N_{L_{i}}\right)$, position angle $\left(\theta_{i}\right)$, and azimuthal width $\left(\sigma_{t_{i}}\right)$. The torus is also allowed to vary in $x_{0}, y_{0}, r_{0}$ and $\sigma_{r 0}$. The lobes are then multiplied by the torus which puts them at a radius of $r_{0}$ and a radial width of $\sigma_{r 0}$, taking the form:

$$
L_{i}=N_{L_{i}} \exp \left[\frac{-\left(\theta-\theta_{i}\right)^{2}}{2 \sigma_{t_{i}}}\right]
$$

where $i$ represents each individual lobe. We combine the torus and the lobes as:

$$
M_{\text {2lobes }}=M_{\text {torus }}\left(1+L_{1}+L_{2}\right)
$$

An example of the fit to this model is shown in Figure $3 \mathrm{~d}$.

\subsection{Quadrilateral Lobes + Torus Model}

A comparison of Figure $3 \mathrm{~b}$ and $3 \mathrm{~d}$ (bilateral model) clearly shows that the bilateral model fails to reproduce the 4 major hot spots seen in the X-ray image. Because there appears to be one such hot spot in each quadrant of the image, we next attempt to model the data using 4 tangentially extended lobes superposed on the torus described in 4.1 with one lobe in each image quadrant (NW, NE, SE, SW). However they are allowed to vary in intensity $\left(N_{t_{i}}\right)$, position angle $\left(\theta_{i}\right)$ within the quadrant, and azimuthal width $\left(\sigma_{t_{i}}\right)$, where $i$ represents the properties of each individual lobes. The torus is also allowed to vary in $x_{0}$, $y_{0}, r_{0}$ and $\sigma_{r 0}$. The lobes are then multiplied by the torus which puts them at a radius of $r_{0}$ and a radial width of $\sigma_{r 0}$, taking the forms of Equation 3. We combine the torus and the lobes as:

$$
M_{\text {4lobes }}=M_{\text {torus }}\left(1+L_{1}+L_{2}+L_{3}+L_{4}\right)
$$

An example of the fit to this model is shown in Figure 3 f.

\section{Results}

We fit the deprojected images from each epoch to each of the three models using a Levenberg-Marquardt two-dimensional least-squares IDL fit procedure. Our models are able to sufficiently represent the data in all cases, with improved reduced $\chi^{2}$ as model complexity increases. Example image fits to all models for sample observation 11 are presented in Figure 3. 
The main goal of this study is to measure the expansion rate of the remnant and follow its evolution. We use the parameter $r_{0}$ measured in each model fit to characterize this behavior. We convert $r_{0}$ into physical velocity units using the distance to SNR 1987A (51.4 $\pm 1.2 \mathrm{kpc}$, Panagia 2003). The expansion measures do not increase linearly throughout, but rather are better fit by a linear fit that extends until $\sim 6000$ days after the $\mathrm{SN}$, and then turns over to a shallower slope (Table 2, Figure 4). Table 3 lists the resulting radial expansion fit parameters for each set of the image model fits. All three models provide consistent fits to the radial expansion measurements with the tightest constrains on $r_{0}$ from the model that best represents the images (quadrilateral lobes plus torus). The results of these fits are shown Figure 4 .

Our estimates of the radial expansion rates before day 6000 are $\sim 65 \%$ larger than previous estimates (Park et al. 2004). This is most likely due to the two different methods used to measure the radius of the SNR. Our radius estimates use more sophisticated image model fits than the simple Gaussian radial profile of the X-ray ring used by the previous work, and thus should be more realistic and reliable. We also considered the $43^{\circ}$ inclination effect of the inner ring causing it to appear elliptical, whereas our previous work assumed a simple circular geometry. For comparison, if we re-incline the measured radial expansion rate, the corresponding expansion velocity is $\sim 5500_{-1100}^{+1600} \mathrm{~km} \mathrm{~s}^{-1}$ prior to day $\sim 6000$ and $\sim$ $1200_{-440}^{+460} \mathrm{~km} \mathrm{~s}^{-1}$ afterwards. This is in plausible agreement with the previous measurements. Hydrodynamic models indicate a forward shock velocity of $\sim 3500-4500 \mathrm{~km} \mathrm{~s}^{-1}$ until day $\sim$ 6000 , and then a dramatic deceleration down to $\sim 1000-1500 \mathrm{~km} \mathrm{~s}^{-1}$ afterwards (Dwarkadas 2007). This overall change of the shock velocity is also in good agreement with the results from our expansion rate estimates.

The most recent radio radial expansion measurements by $\mathrm{Ng}$ et al. (2008) show a constant remnant expansion rate of $4000 \pm 400 \mathrm{~km} \mathrm{~s}^{-1}$ over a range of 1992-2008. However, the radio remnant radius is systematically larger than the X-ray remnant radius by $\sim 10-20 \%$. Interestingly, after day $\sim 5400$, the radio remnant radius is larger than the radius of the optical inner ring (0".83, Sugerman et al. 2002). This discrepancy in the SNR size between the X-ray and radio data is largely caused by the different image modeling techniques as discussed by $\mathrm{Ng}$ et al. (2008). It may also indicate that the X-ray and radio emission are produced in different physical regions. The radio emission is likely due to synchrotron processes in between the forward and reverse shocks (Manchester et al. 2005), while the X-rays are dominated by thermal emission from the transmitted and reflected shocks produced by the shock-inner ring interaction (Zhekov et al. 2009). The 3-dimensional effects and emission regions also likely differ between these components. Ng et al. (2008) predict that they will soon see a deceleration in the radio remnant expansion similar to that we have observed from the X-ray remnant due to the forward shock interacting with the optical inner ring. 


\section{Exploring Methods with Simulated Images}

In order to test the reliability of our image modeling and radial expansion measures, we created a series of simulated images using the Model of AXAF Response to X-rays (MARX) tool. The simulated images were meant to approximate the real images obtained at days $\sim 4600,5200,6200$, and 7200 days after the SN explosion, assuming the estimated radius for each epoch. They were generated with different observed inclinations and properties, specifically 0 degrees inclination (face-on), and 45 degrees inclination both with and without 4 spots. We applied the Chandra PSF to the simulated images to mimic observing effects, and ran them through the same image deconvolution process as done with the real data.

We processed these simulated images through the same algorithms used to fit the observed images for all of our image models. The results showed a systematic offset in the best-fit radius, which is $\sim 10 \%$ smaller than the assumed input values, yet gave an accurate measure of the radial expansion rate within measurement errors. We attribute this systematic offset to differences in the treatment of the torus width $\left(\sigma_{r_{0}}\right)$. The simulated images were constructed assuming that the torus and any changes to the width over time are not spatially resolved. Applying this same assumption to the simulation fits, we froze the torus width to approximately the value of the effective PSF for the deconvolved images $\left(\sim 0^{\prime \prime} .2-0^{\prime \prime} \cdot 3\right)$, and redid the fits. This effectively eliminated the systematic offset and reproduced the input radii accurately.

In the application to the real data, when leaving the torus width as a free parameter, the model fits may underestimate the radius of the real remnant images by as much as

$10 \%$. However, the free torus width fits appear to reveal a widening torus with values at a similar size to the PSF. Any real physical changes to the torus width would be ignored if the parameter is frozen to a set value. The width is not independent of the radii measurement that we are inherently trying to measure. The fits to the real data are statistically improved if we assume that the torus has some real width that may change over time and the width parameter is left free to vary. The fitted torus width (Gaussian half-width) ranges from $\sim 0^{\prime \prime} .12-0^{\prime \prime} .21$ which is significantly smaller than the ring radius and comparable to the effective PSF of the deconvolved Chandra images.

We also assume a 2-dimensional structure in all of our model fitting even with the deprojecting correction. In reality, X-ray emission most likely originates from a 3-dimensional structure with the shock component being perpendicular to the disk containing the inner ring, making contributions to the torus width unknown. The real 3-dimensional geometry of the unresolved inner ring is not yet well understood. The potential $\sim 10 \%$ radii offset is systematic and may consequently have the same effect on the expansion velocities. However, the measurement errors are larger than this systematic offset. 
The first two images are most strongly affected by freezing the torus width because they suffer from low count statistics (lower by an order of magnitude than those at other epochs), and contain the lowest contributions of the X-ray emission from the shock-inner ring interaction. Therefore, this 3-dimensional effect could have been relatively large in these early images. To test this effect, we fit the radial expansion rate using the frozen torus width with and without the first two images, with the latter analysis leading to a more accurate reproduction of the results from the free torus width fits. When including the first two observations with a frozen torus radius $\left(r_{0} \sim 0.15^{\prime \prime}\right)$, the initial expansion velocity is $\sim 12000 \mathrm{~km} \mathrm{~s}^{-1}$, flattening to a velocity of $\sim 1400 \mathrm{~km} \mathrm{~s}^{-1}$ at $\sim 5800$ days. The expansion measurement excluding the first two observations yields an initial expansion velocity of $\sim 6000 \mathrm{~km} \mathrm{~s}^{-1}$ flattening to a velocity of $\sim 1700 \mathrm{~km} \mathrm{~s}^{-1}$ at $\sim 6200$ days. Therefore, it is these early images which are influencing the fits and are least conducive to a frozen torus width. Even with a free torus width, the first two images are the noisiest and provide the poorest fits. The first two images have some effect on the accuracy of the expansion rate, but do not change the overall conclusion that we observe a slowing of the expansion rate starting at $\sim 6000$ days.

\section{Conclusions}

Multi-wavelength studies at frequent epochs are carefully capturing the evolution and progression of SNR 1987A. As the evolution continues, it reveals new regions of emission and probes a larger volume of the circumstellar environment. We believe that the predicted events (Chevalier \& Liang 1989; Chevalier \& Dwarkadas 1995; Luo \& McCray 1991; Borkowski et al. 1997) of the SN blast wave engulfing the inner circumstellar ring are occurring. The earlier optical and soft X-ray emission spots were a result of higher density gas protruding inward from the main ring. In recent years, we have seen the entire ring begin to light up. The current optical and X-ray emission is a composite of the shocks heating the higher and lower density regions of the clumpy ring structure.

Our measurement of the deceleration of the radial expansion of SNR 1987A from $\sim 8000 \mathrm{~km} \mathrm{~s}^{-1}$ to $\sim 1600 \mathrm{kms}^{-1}$ at $\sim 6000$ days along with the upturn of the X-ray luminosity, fractional contribution from the decelerated shock to the soft X-ray flux, and the low shock velocities from line broadening are all pointing to indications of the SN blast wave encountering the main body of the circumstellar ring around day 6200. The evidence of the blast wave encountering the inner ring are already apparent in the X-ray observations, and we are yet to see the strong indications from the radio or optical observations. The details of the different emission regions and their interactions with the different shock components 
will become clearer as continued optical, X-ray, and radio observations probe the remnant.

Continued monitoring of SNR 1987A with Chandra will reveal more details in the structure of the circumstellar environment around the remnant. We expect to see additional brightening as the blast wave shock front heats the material in the ring and as the reverse shock region continues to grow in volume. As the remnant expands and brightens, we hope to further resolve structures and to understand the inhomogeneous nature of the circumstellar environment. We expect the expansion to continue to slow as it sweeps up more material and transitions from the free expansion phase to the adiabatic expansion phase once it has swept up a mass of material comparable to the SN ejecta. Uncertainties in this ejecta mass make predictions for this time frame difficult. However, SNR 1987A has an unusually high density environment as evident by the effects on the original SN explosion. Therefore this transition may occur earlier than the typical $\sim 200$ year timescale.

We may also expect to see additional deceleration of the radial expansion while the blast wave sweeps up more of the circumstellar material. Only the material that was photo-ionized by the UV flash from the SN is visible in the optical inner ring, and there is evidence suggesting that there may be more mass that was not ionized (Crotts et al. 1989; Ensman \& Burrows 1992). The inner ring has an ionized mass of $\sim 0.04 \mathrm{M}_{\odot}$, which is probably only the inner surface of a larger gas complex. As the hot spots brighten and produce ionizing radiation, eventually the undetected outer material will become visible as an emission nebula (McCray 2003). The UV and optical light echoes seen several months after the SN event hinted at the structure of the dust in the outer circumstellar environment. It will be interesting to continue to use the radial expansion measurement to probe the density structure of the ring.

This work is supported by Chandra SAO grants GO7-8069X and GO8-9076X. S.P. was also supported in part by SAO grant SV4-74018. 


\section{REFERENCES}

Arnett, W. D., Bahcall, J. N., Kirshner, R. P., \& Woosley, S. E. 1989, ARA\&A, 27, 629

Borkowski, K. J., Blondin, J. M., \& McCray, R. 1997, ApJ, 477, 281

Bouchet, P., Dwek, E., Danziger, J., Arendt, R. G., De Buizer, I. J. M., Park, S., Suntzeff, N. B., Kirshner, R. P., \& Challis, P. 2006, ApJ, 650, 212

Burrows, C. J., Krist, J., Hester, J. J., Sahai, R., Trauger, J. T., Stapelfeldt, K. R., Gallagher, III, J. S., Ballester, G. E., Casertano, S., Clarke, J. T., Crisp, D., Evans, R. W., Griffiths, R. E., Hoessel, J. G., Holtzman, J. A., Mould, J. R., Scowen, P. A., Watson, A. M., \& Westphal, J. A. 1995, ApJ, 452, 680

Burrows, D. N., Michael, E., Hwang, U., McCray, R., Chevalier, R. A., Petre, R., Garmire, G. P., Holt, S. S., \& Nousek, J. A. 2000, ApJ, 543, L149

Chevalier, R. A., \& Dwarkadas, V. V. 1995, ApJ, 452, L45

Chevalier, R. A., \& Liang, E. P. 1989, ApJ, 344, 332

Crotts, A. P., \& Heathcote, S. R. 1991, Nature, 350, 683

Crotts, A. P. S., Kunkel, W. E., \& McCarthy, P. J. 1989, ApJ, 347, L61

Dewey, D., Zhekov, S. A., McCray, R., \& Canizares, C. R. 2008, ApJ, 676, L131

Dwarkadas, V. V. 2007, in American Institute of Physics Conference Series, Vol. 937, American Institute of Physics Conference Series, ed. S. Immler \& R. McCray, 120-124

Ensman, L., \& Burrows, A. 1992, ApJ, 393, 742

Gaensler, B. M., Manchester, R. N., Staveley-Smith, L., Tzioumis, A. K., Reynolds, J. E., \& Kesteven, M. J. 1997, ApJ, 479, 845

Lucy, L. B. 1974, AJ, 79, 745

Luo, D., \& McCray, R. 1991, ApJ, 372, 194

Manchester, R. N., Gaensler, B. M., Staveley-Smith, L., Kesteven, M. J., \& Tzioumis, A. K. 2005, ApJ, 628, L131

Manchester, R. N., Gaensler, B. M., Wheaton, V. C., Staveley-Smith, L., Tzioumis, A. K., Bizunok, N. S., Kesteven, M. J., \& Reynolds, J. E. 2002, Publications of the Astronomical Society of Australia, 19, 207 
McCray, R. 2003, in Lecture Notes in Physics, Berlin Springer Verlag, Vol. 598, Supernovae and Gamma-Ray Bursters, ed. K. Weiler, 219-240

McCray, R. 2005, in IAU Colloq. 192: Cosmic Explosions, On the 10th Anniversary of SN1993J, ed. J.-M. Marcaide \& K. W. Weiler, 77

Michael, E., Zhekov, S., McCray, R., Hwang, U., Burrows, D. N., Park, S., Garmire, G. P., Holt, S. S., \& Hasinger, G. 2002, ApJ, 574, 166

Mori, K., Tsunemi, H., Miyata, E., Baluta, C. J., Burrows, D. N., Garmire, G. P., \& Chartas, G. 2001, in Astronomical Society of the Pacific Conference Series, Vol. 251, New Century of X-ray Astronomy, ed. H. Inoue \& H. Kunieda, 576

Ng, C.-Y., Gaensler, B. M., Staveley-Smith, L., Manchester, R. N., Kesteven, M. J., Ball, L., \& Tzioumis, A. K. 2008, ApJ, 684, 481

Panagia, N. 2003, ArXiv Astrophysics e-prints

Park, S., Burrows, D. N., Garmire, G. P., Nousek, J. A., McCray, R., Michael, E., \& Zhekov, S. 2002, ApJ, 567, 314

Park, S., Zhekov, S. A., Burrows, D. N., Garmire, G. P., \& McCray, R. 2004, ApJ, 610, 275

Park, S., Zhekov, S. A., Burrows, D. N., Garmire, G. P., Racusin, J. L., \& McCray, R. 2006a, ApJ, 646, 1001

Park, S., Zhekov, S. A., Burrows, D. N., \& McCray, R. 2005, ApJ, 634, L73

Park, S., Zhekov, S. A., Burrows, D. N., Racusin, J. L., McCray, R., \& Borkowski, K. J. 2006b, in ESA Special Publication, Vol. 604, The X-ray Universe 2005, ed. A. Wilson, $335-340$

Plait, P. C., Lundqvist, P., Chevalier, R. A., \& Kirshner, R. P. 1995, ApJ, 439, 730

Richardson, W. H. 1972, Journal of the Optical Society of America (1917-1983), 62, 55

Sonneborn, G., Pun, C. S. J., Kimble, R. A., Gull, T. R., Lundqvist, P., McCray, R., Plait, P., Boggess, A., Bowers, C. W., Danks, A. C., Grady, J., Heap, S. R., Kraemer, S., Lindler, D., Loiacono, J., Maran, S. P., Moos, H. W., \& Woodgate, B. E. 1998, ApJ, 492, L139

Sugerman, B. E. K., Lawrence, S. S., Crotts, A. P. S., Bouchet, P., \& Heathcote, S. R. 2002, ApJ, 572, 209 
Townsley, L. K., Broos, P. S., Garmire, G. P., \& Nousek, J. A. 2000, ApJ, 534, L139

Tsunemi, H., Mori, K., Miyata, E., Baluta, C., Burrows, D. N., Garmire, G. P., \& Chartas, G. 2001, ApJ, 554, 496

White, G. L., \& Malin, D. F. 1987, Nature, 327, 36

Zhekov, S. A., McCray, R., Borkowski, K. J., Burrows, D. N., \& Park, S. 2005, ApJ, 628, L127

-. 2006, ApJ, 645, 293

Zhekov, S. A., McCray, R., Dewey, D., Canizares, C. R., Borkowski, K. J., Burrows, D. N., \& Park, S. 2009, ApJ, 692, 1190 
Table 1. Chandra Observations of SNR 1987A

\begin{tabular}{|c|c|c|c|c|c|}
\hline Observation \# & Obs Date & Age* $^{*}$ & Instrument $^{\diamond}$ & $\begin{array}{c}\text { Exposure } \\
(\mathrm{ks})\end{array}$ & $\begin{array}{l}\text { Source } \\
\text { Counts }\end{array}$ \\
\hline 1 & 1999 October 6 & 4608 & ACIS-S + HETG ${ }^{\dagger}(3.1 \mathrm{~s})$ & 116.1 & 690 \\
\hline 2 & 2000 January 17 & 4711 & ACIS-S3 $(3.2 \mathrm{~s})$ & 8.6 & 607 \\
\hline 3 & 2000 December 7 & 5036 & ACIS-S3 $(3.2 \mathrm{~s})$ & 98.8 & 9030 \\
\hline 4 & 2001 April 25 & 5175 & ACIS-S3 $(3.2 \mathrm{~s})$ & 17.8 & 1800 \\
\hline 5 & 2001 December 12 & 5406 & ACIS-S3 $(3.1 \mathrm{~s})$ & 49.4 & 6226 \\
\hline 6 & 2002 May 5 & 5560 & ACIS-S3 $(3.1 \mathrm{~s})$ & 44.3 & 6427 \\
\hline 7 & 2002 December 31 & 5790 & ACIS-S3 $(3.1 \mathrm{~s})$ & 49.0 & 9277 \\
\hline 8 & 2003 July 8 & 5979 & ACIS-S3 $(3.1 \mathrm{~s})$ & 45.3 & 9668 \\
\hline 9 & 2004 January 2 & 6157 & ACIS-S3 $(3.1 \mathrm{~s})$ & 46.5 & 11856 \\
\hline 10 & 2004 July 22 & 6359 & ACIS-S3 $(1.5 \mathrm{~s})$ & 48.8 & 17979 \\
\hline 11 & 2005 January 12 & 6532 & ACIS-S3 $(0.4 \mathrm{~s})$ & 48.3 & 24939 \\
\hline 12 & 2005 July 14 & 6716 & ACIS-S3 $(0.4 \mathrm{~s})$ & 44.1 & 27048 \\
\hline 13 & 2006 January 28 & 6914 & ACIS-S3 $(0.4 \mathrm{~s})$ & 42.3 & 30940 \\
\hline 14 & 2006 July 28 & 7094 & ACIS-S3 $(0.4 \mathrm{~s})$ & 36.4 & 30870 \\
\hline 15 & 2007 January 19 & 7270 & ACIS-S3 $(0.4 \mathrm{~s})$ & 33.5 & 32798 \\
\hline 16 & 2007 July 13 & 7445 & ACIS-S3 $(0.4 \mathrm{~s})$ & 25.7 & 27945 \\
\hline 17 & 2008 January 10 & 7626 & ACIS-S3 $(0.2 \mathrm{~s})$ & 9.3 & 12008 \\
\hline 18 & 2008 July 4 & 7802 & ACIS-S3 $(0.2 \mathrm{~s})$ & 8.6 & 12119 \\
\hline 19 & 2009 January 5 & 7987 & ACIS-S3 $(0.2 \mathrm{~s})$ & 6.0 & 9204 \\
\hline
\end{tabular}

${ }^{*}$ Days since SN explosion

$\dagger$ Zeroth order data from grating observations

$\ddagger 0.3-8.0 \mathrm{keV}$ band

${ }^{\bullet}$ Time in parentheses is the ACIS frame-time. 
Table 2. Model Parameter Fits

\begin{tabular}{ccccccc}
\hline \hline \multirow{2}{*}{ Age $^{*}$} & \multicolumn{2}{c}{ Torus Only } & \multicolumn{2}{c}{ Torus +2 lobes } & \multicolumn{2}{c}{ Torus+4 lobes } \\
& $\sigma_{r_{0}}(\operatorname{arcsec})$ & $r_{0}(\operatorname{arcsec})$ & $\sigma_{r_{0}}(\operatorname{arcsec})$ & $r_{0}(\operatorname{arcsec})$ & $\sigma_{r_{0}}(\operatorname{arcsec})$ & $r_{0}(\operatorname{arcsec})$ \\
\hline \multirow{2}{*}{4608} & $0.12 \pm 0.01$ & $0.60 \pm 0.02$ & $0.12 \pm 0.01$ & $0.60 \pm 0.02$ & $0.12 \pm 0.01$ & $0.60 \pm 0.02$ \\
4711 & $0.14 \pm 0.01$ & $0.59 \pm 0.02$ & $0.14 \pm 0.01$ & $0.59 \pm 0.02$ & $0.14 \pm 0.01$ & $0.59 \pm 0.02$ \\
5036 & $0.19 \pm 0.01$ & $0.65 \pm 0.01$ & $0.18 \pm 0.01$ & $0.66 \pm 0.01$ & $0.18 \pm 0.01$ & $0.66 \pm 0.01$ \\
5175 & $0.17 \pm 0.01$ & $0.66 \pm 0.02$ & $0.16 \pm 0.01$ & $0.64 \pm 0.02$ & $0.17 \pm 0.01$ & $0.67 \pm 0.02$ \\
5406 & $0.19 \pm 0.01$ & $0.68 \pm 0.01$ & $0.19 \pm 0.01$ & $0.68 \pm 0.01$ & $0.19 \pm 0.01$ & $0.69 \pm 0.01$ \\
5560 & $0.19 \pm 0.01$ & $0.68 \pm 0.01$ & $0.19 \pm 0.01$ & $0.67 \pm 0.01$ & $0.19 \pm 0.01$ & $0.69 \pm 0.01$ \\
5790 & $0.22 \pm 0.01$ & $0.69 \pm 0.01$ & $0.22 \pm 0.01$ & $0.71 \pm 0.01$ & $0.22 \pm 0.01$ & $0.72 \pm 0.01$ \\
5979 & $0.20 \pm 0.01$ & $0.73 \pm 0.01$ & $0.21 \pm 0.01$ & $0.72 \pm 0.01$ & $0.21 \pm 0.01$ & $0.73 \pm 0.01$ \\
6157 & $0.22 \pm 0.01$ & $0.73 \pm 0.01$ & $0.22 \pm 0.01$ & $0.73 \pm 0.01$ & $0.22 \pm 0.01$ & $0.74 \pm 0.01$ \\
6359 & $0.22 \pm 0.01$ & $0.74 \pm 0.01$ & $0.22 \pm 0.01$ & $0.74 \pm 0.01$ & $0.22 \pm 0.01$ & $0.74 \pm 0.01$ \\
6532 & $0.22 \pm 0.01$ & $0.74 \pm 0.01$ & $0.21 \pm 0.01$ & $0.74 \pm 0.01$ & $0.21 \pm 0.01$ & $0.74 \pm 0.01$ \\
6716 & $0.21 \pm 0.01$ & $0.75 \pm 0.01$ & $0.21 \pm 0.01$ & $0.74 \pm 0.01$ & $0.21 \pm 0.01$ & $0.75 \pm 0.01$ \\
6914 & $0.22 \pm 0.01$ & $0.74 \pm 0.01$ & $0.22 \pm 0.01$ & $0.74 \pm 0.01$ & $0.22 \pm 0.01$ & $0.75 \pm 0.01$ \\
7094 & $0.21 \pm 0.01$ & $0.76 \pm 0.01$ & $0.21 \pm 0.01$ & $0.75 \pm 0.01$ & $0.21 \pm 0.01$ & $0.76 \pm 0.01$ \\
7270 & $0.21 \pm 0.01$ & $0.76 \pm 0.01$ & $0.21 \pm 0.01$ & $0.76 \pm 0.01$ & $0.21 \pm 0.01$ & $0.76 \pm 0.01$ \\
7445 & $0.20 \pm 0.01$ & $0.77 \pm 0.01$ & $0.21 \pm 0.01$ & $0.77 \pm 0.01$ & $0.21 \pm 0.01$ & $0.77 \pm 0.01$ \\
7626 & $0.21 \pm 0.01$ & $0.76 \pm 0.01$ & $0.21 \pm 0.01$ & $0.76 \pm 0.01$ & $0.21 \pm 0.01$ & $0.76 \pm 0.01$ \\
7802 & $0.20 \pm 0.01$ & $0.77 \pm 0.01$ & $0.20 \pm 0.01$ & $0.76 \pm 0.01$ & $0.20 \pm 0.01$ & $0.76 \pm 0.01$ \\
7987 & $0.20 \pm 0.01$ & $0.78 \pm 0.01$ & $0.20 \pm 0.01$ & $0.78 \pm 0.01$ & $0.20 \pm 0.01$ & $0.78 \pm 0.01$ \\
\hline & & & & & & \\
\hline
\end{tabular}

*Days since SN explosion

Note. - Errors are $1 \sigma$ confidence intervals. 
Table 3. Radial Expansion Velocity Fits

\begin{tabular}{ccccc}
\hline \hline & $\begin{array}{c}v_{a} \\
\left(\mathrm{~km} \mathrm{~s}^{-1}\right)\end{array}$ & $\begin{array}{c}\text { turn over time } \\
(\text { days })\end{array}$ & $\begin{array}{c}v_{b} \\
\left(\mathrm{~km} \mathrm{~s}^{-1}\right)\end{array}$ & $\chi_{v_{a b}}^{2} /$ dof \\
\hline Torus only & $6908_{-1127}^{+1283}$ & $6184_{-220}^{+256}$ & $1961_{-635}^{+602}$ & 0.70 \\
Torus + 2 Lobes & $7047_{-1204}^{+1460}$ & $6135_{-284}^{+208}$ & $2037_{-578}^{+532}$ & 0.53 \\
Torus + 4 Lobes & $7539_{-1524}^{+2139}$ & $6060_{-237}^{+208}$ & $1592_{-566}^{+589}$ & 0.54 \\
\hline
\end{tabular}

Note. - $v_{a}$ and $v_{b}$ are calculated using the slopes of the broken linear fit with 15 DOF. All errors are $90 \%$ confidence. 

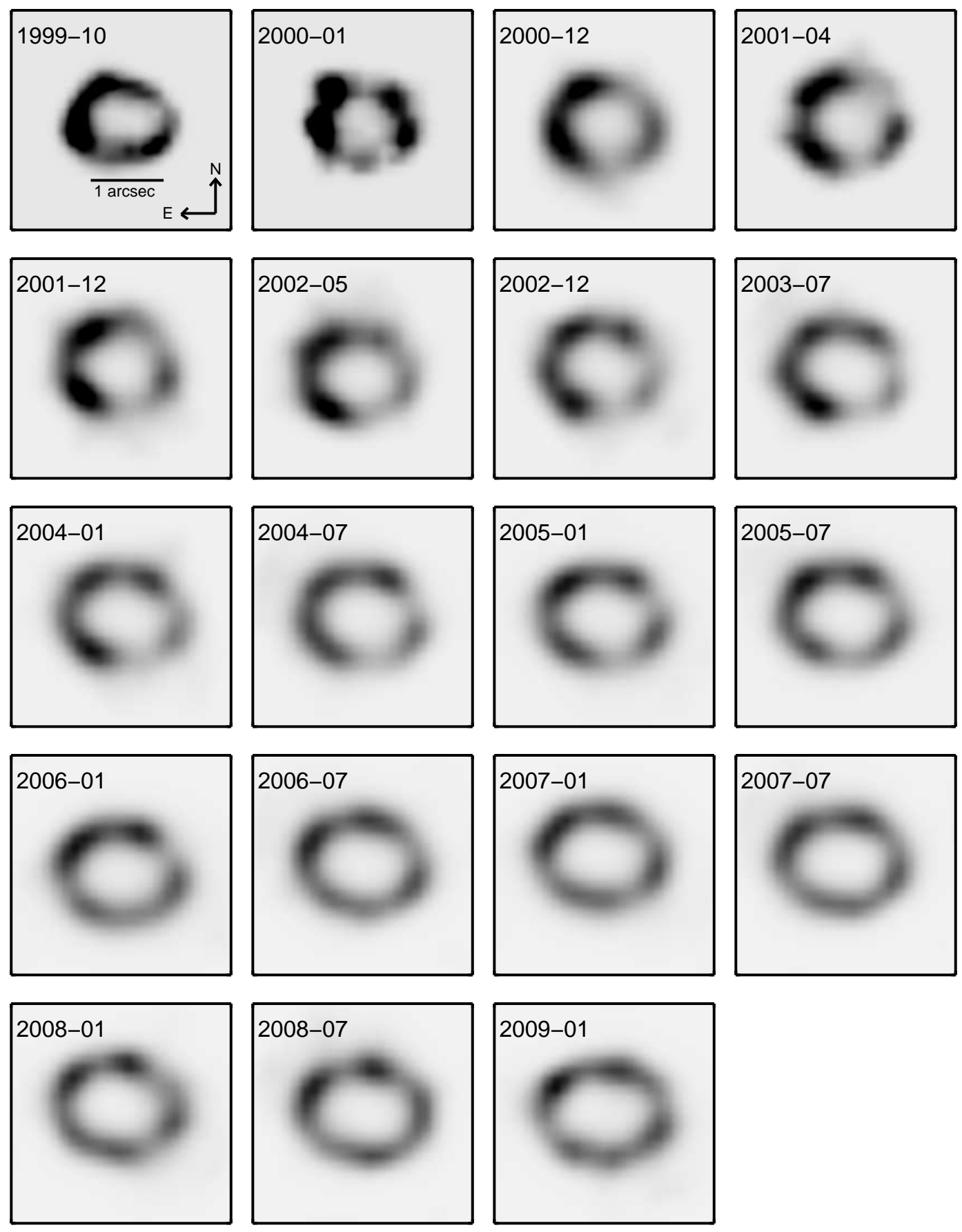

Fig. 1.- Broadband $(0.3-8.0 \mathrm{keV})$ images of SNR 1987A from each Chandra observation taken on date (YYYY-MM) as labeled. The images are all the same spatial scale with slight uncertainty in rotations and centers due to unknown absolute astrometry on these scales. The images are scaled arbitrarily to show the morphological details of the evolving remnant. The flux has actually increased dramatically over the course of this monitoring campaign, as shown in Figure 2. 


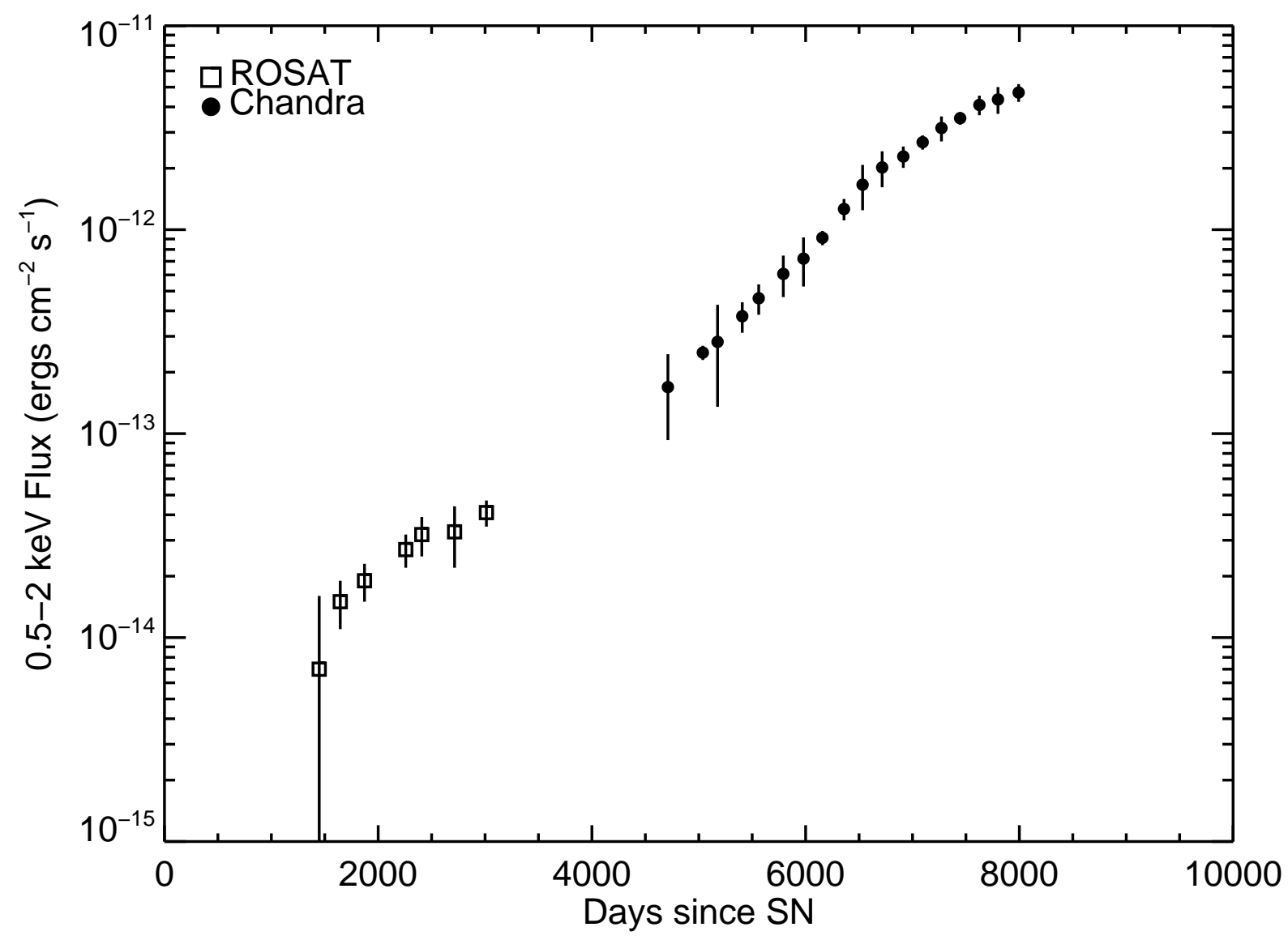

Fig. 2.- Soft X-ray $(0.5-2 \mathrm{kev})$ light curve from ROSAT and Chandra observations. 

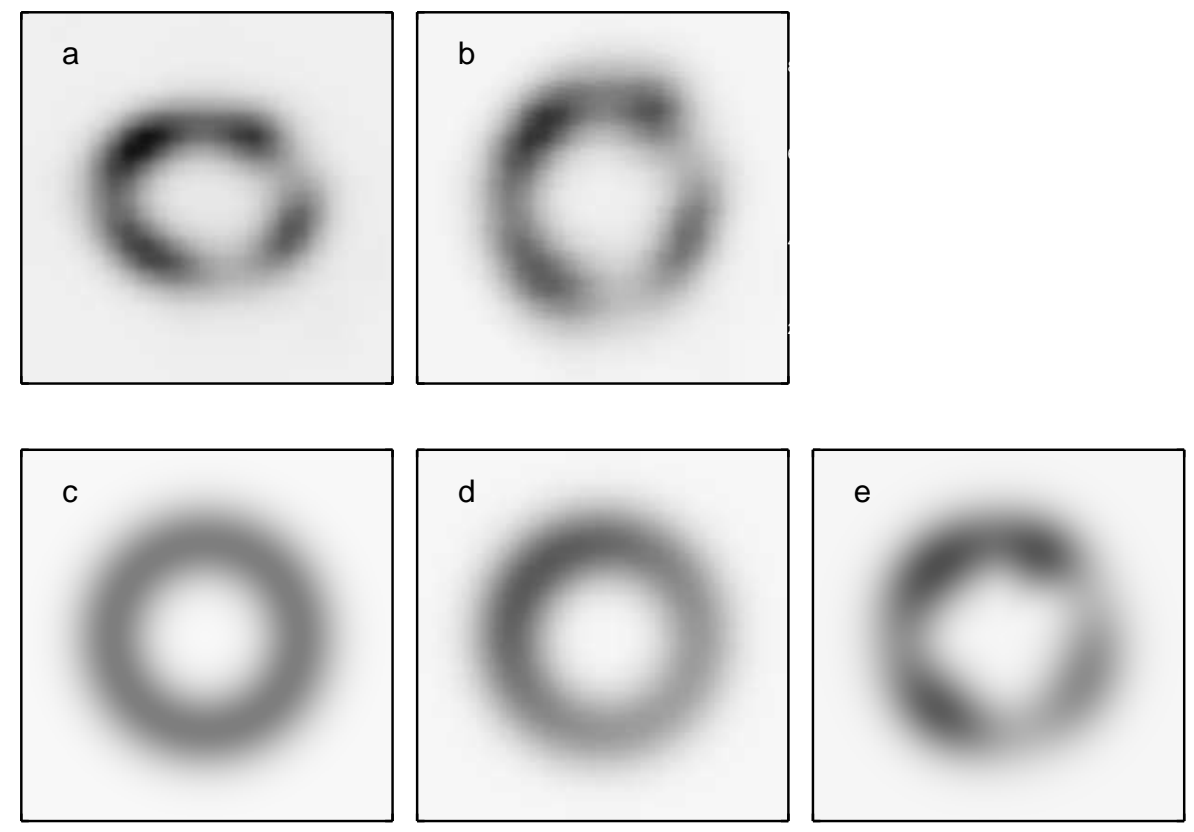

Fig. 3.- Images and fits to models for observation 11. a: original deconvolved sub-pixel image, $b$ : deprojected image, $c$ : fit to torus only model, $d$ : fit to the torus plus two-lobes model, $e$ : fit to torus plus four-lobes model. 


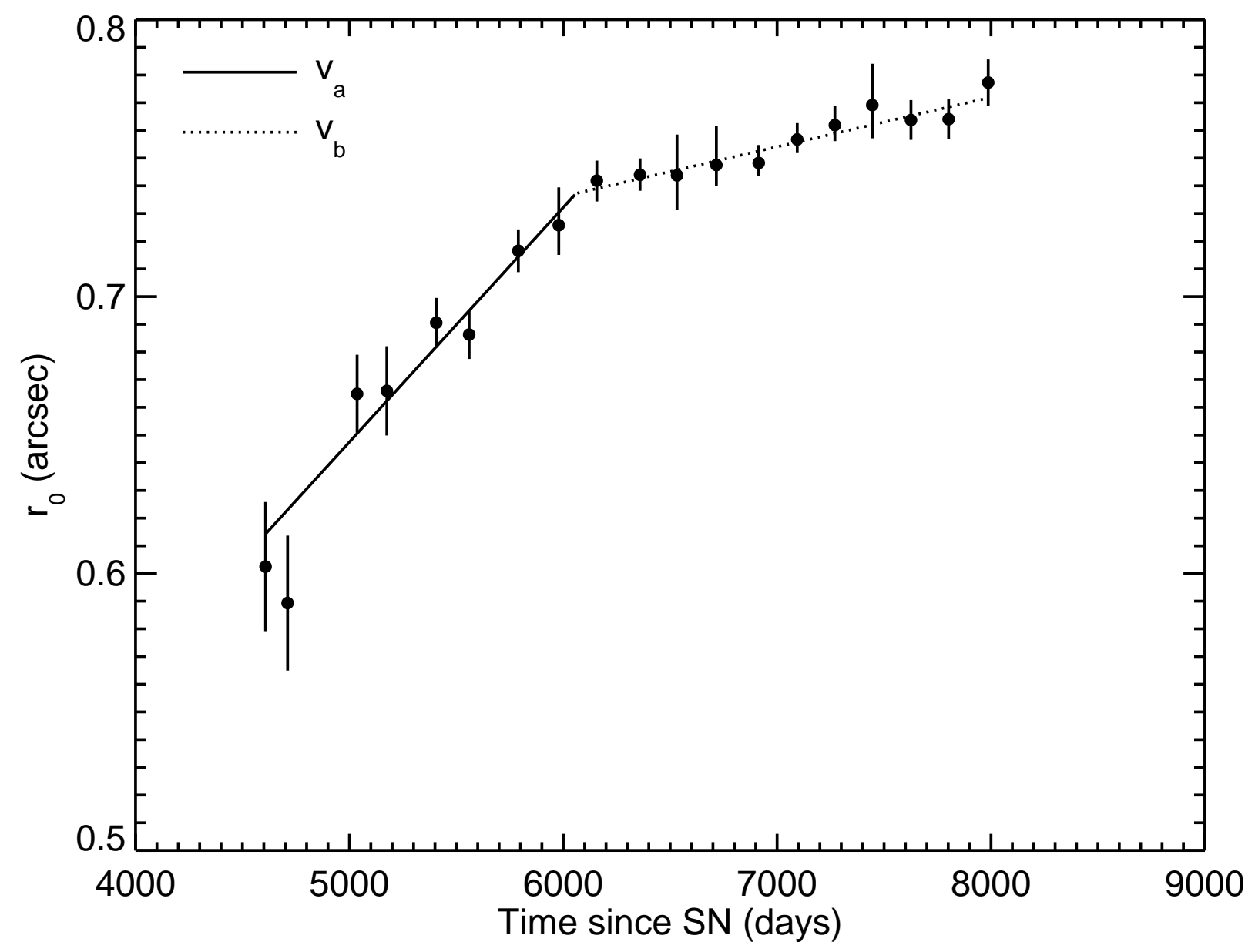

Fig. 4.- Radial expansion measure from the fits to the quadrilateral lobes plus torus model for the broken linear fit with an early fast shock velocity $\left(v_{a}\right)$ transitioning to the later slower shock velocity $\left(v_{b}\right)$. The radii for the 3 image models are listed in Table 2, and the resulting radial expansion fits are given in Table 3 . 